\title{
Vector-valued Coherent Risk Measures
}

\author{
E. Jouini $^{*} \quad$ M. Meddeb ${ }^{\dagger} \quad$ N. Touzi ${ }^{\ddagger}$
}

This version : December 2002

\begin{abstract}
We define $(d, n)$-coherent risk measures as set-valued maps from $L_{d}^{\infty}$ into $\mathbb{R}^{n}$ satisfying some axioms. We show that this definition is a convenient extension of the real-valued risk measures introduced by Artzner, Delbaen, Eber and Heath (1998). We then discuss the aggregation issue, i.e. the passage from $\mathbb{R}^{d}$-valued random portfolio to $\mathbb{R}^{n}$-valued measure of risk. Necessary and sufficient conditions of coherent aggregation are provided.
\end{abstract}

\section{Introduction}

The concept of coherent risk measures together with its axiomatic characterization was introduced by Artzner, Delbaen, Eber and Heath (1998, ADEH) in a finite probability space, and further extended by Delbaen (2000) to the general probability space framework. In the above mentioned papers, the risky portfolio under consideration is a given real-valued random variable. A risk measure $\rho$ is then defined as a map from $L^{\infty}$ into $\mathbb{R}$ satisfying some coherency axioms, so that for any $X \in L^{\infty}, \rho(X+\rho(X))=0$, i.e. the deterministic amount $\rho(X)$ cancels the risk of $X$. The extension of this map to unbounded random variables is discussed in Delbaen [?]. In this paper, we focus on the more realistic situation where the risky portfolio is an $\mathbb{R}^{d}$-valued random variable. We assume that a partial ordering $\succeq$ on $\mathbb{R}^{d}$ is given. The specification of $\succeq$ accounts for some frictions on the financial market such as transaction costs, liquidity problems, irreversible transfers, etc... We first provide an extension of the axiomatic characterization of ADEH to this multi-dimensional framework. Given an integer $n \leq d$, we define $(d, n)$-coherent risk measure (consistent with $\succeq$ ) as a setvalued map $R$ from $L_{d}^{\infty}$ into $\mathbb{R}^{n}$ satisfying some convenient axioms. When $n=d=1$, we recover the results of [?] by setting $R=[\rho, \infty)$. Our definition of vector-valued coherent risk measures allows for $n<d$, i.e. the risk of the $\mathbb{R}^{d}$-valued random variable is required to be

${ }^{*}$ CEREMADE, Université Paris Dauphine, and CREST, jouini@ceremade.dauphine.fr

${ }^{\dagger}$ CERMSEM, Université Paris Panthéon-Sorbonne, meddeb@univ-paris1.fr

${ }^{\ddagger}$ CREST and CEREMADE, touzi@ensae.fr 
cancelled by $\mathbb{R}^{n}$-deterministic portfolios. This is related to an aggregation problem. The second part of this paper then concentrates on this issue. We study two alternative methods of aggregation. (i) portfolio aggregation : Given an $(n, n)$-coherent risk measure, and a deterministic function $f: \mathbb{R}^{d} \longrightarrow \mathbb{R}^{n}$, we provide necessary and sufficient conditions for the set-valued map $R \circ f$ to be a $(d, n)$-coherent risk measure, (ii) risk aggregation : Given a $(d, d)$-coherent risk measure, and a deterministic function $g: \mathbb{R}^{d} \longrightarrow \mathbb{R}^{n}$, we provide necessary and sufficient conditions for the set-valued map $\operatorname{cl}[g \circ R]$ to be a $(d, n)$-coherent risk measure. The paper is organized as follows. Section ?? contains our definition of vectorvalued coherent risk measure. Section ?? reports the geometric and topological properties implied by the suggested definition, and needed for the subsequent analysis. Section ?? provides the dual representation of vector-valued coherent risk measures. In section ??, we discuss the problem of relevancy, a notion which is closely related to the no-arbitrage condition. Finally, the above mentioned aggregation issue is addressed in sections ?? and ??. Notations. Throughout this paper, we shall denote by $x_{i}$ the $i$-th component of an

element $x$ of a finite dimensional vector space. We shall denote by $\mathbf{1}^{i}$ the $i$-th canonical basis vector defined by $\mathbf{1}_{j}^{i}=1$ if $i=j$, zero otherwise, and we set $\mathbf{1}:=\sum_{i} \mathbf{1}^{i}$ the vector with unit components. The latter notation should not be confused with the indicator function $\mathbf{1}_{A}$ of a set $A$. The closure, the interior, and the relative interior of a set will be denoted respectively by $\operatorname{cl}[\cdot]$, int[·], and $\operatorname{ri}[\cdot]$. Given a subset $A \subset \mathbb{R}^{d}$, we shall denote by $L_{d}^{p}(A)$ the collection of $A$-valued random variables with finite $L^{p}$-norm. We shall use the simplified notation $L_{d}^{p}:=L_{d}^{p}\left(\mathbb{R}^{d}\right), L^{p}(A):=L_{1}^{p}(A)$, and $L^{p}:=L^{p}(\mathbb{R})$. As usual, $L^{0}$ and $L^{\infty}$ stand respectively for the set of all measurable functions, and all essentially bounded functions.

\section{Vector-valued Coherent Risk Measures on $L_{d}^{\infty}$}

\subsection{The general framework}

Let $(\Omega, \mathcal{F}, \mathbb{P})$ be a probability space. In this paper we study the financial risk induced by a random portfolio from the point of view of the regulator/supervisor. In mathematical words, a (random) portfolio is a vector-valued random variable $X$ on the probability space $(\Omega, \mathcal{F}, \mathbb{P})$. We shall restrict our attention to portfolios in $L_{d}^{\infty}$, the space of all equivalence classes of (essentially) bounded $\mathbb{R}^{d}$-valued random variables. We intend to extend the notion of coherent risk measure to the multi-dimensional case. Real-valued coherent measures of risk have been introduced by ADEH [?]; see also Delbaen [?] for the general probability space setting. 


\subsection{Portfolio ordering}

Portfolios in $L_{d}^{\infty}$ are (partially) ordered according to the following rule. Let $K$ be a closed convex cone of $\mathbb{R}^{d}$ such that

$$
\mathbb{R}_{+}^{d} \subset K \text { and } K \neq \mathbb{R}^{d}
$$

The closed convex cone $K$ induces the partial ordering $\succeq$ on $\mathbb{R}^{d}$ by $x \succeq 0$ iff $x \in K$. We extend naturally the partial ordering $\succeq$ to $L_{d}^{\infty}$ by :

$$
X \succeq 0 \quad \text { iff } \quad X \in K \quad P-\text { a.s. }
$$

With this definition the condition $\mathbb{R}_{+}^{d} \subset K$ means that any portfolio $x$ with non-negative entries is non-negative in the sense of the partial ordering $\succeq$. We assume further that $K$ satisfies the substitutability condition :

(2.2) for all $i=n+1, \ldots, d: \quad-\mathbf{1}^{i}+\alpha \mathbf{1}^{1}$ and $\mathbf{1}^{i}-\beta \mathbf{1}^{1} \in K \quad$ for some $\alpha, \beta>0$.

Condition (??) means that any position on each entry $i>n$ can be compensated by some position on the first entry. More precisely, it states that the unitary prices of the assets $i>n$ in terms of the assets $j \leq n$ must be bounded. In the case $n=d$, condition (??) is empty. Finally, we define the liquidation function:

$$
\ell(x):=\sup \left\{w \in \mathbb{R}: x \succeq w \mathbf{1}^{1}\right\}
$$

which is valued in $\mathbb{R} \cup\{+\infty\}$. From the substitutability condition (??) together with the closedness of $K$, we have :

$$
\ell(x)=\max \left\{w \in \mathbb{R}: x \succeq w \mathbf{1}^{1}\right\}<\infty \text { for all } x \in \mathbb{R} \times\{0\}^{n-1} \times \mathbb{R}^{d-n} .
$$

We shall make use of the notation

$$
\bar{\pi}(x):=\sum_{i=1}^{n} x_{i} \mathbf{1}^{i}+\ell\left(\sum_{i=n+1}^{d} x_{i} \mathbf{1}^{i}\right) \mathbf{1}^{1} \text { for all } x \in \mathbb{R}^{d} .
$$

Observe that the the last $d-n$ components of the $\mathbb{R}^{d}$-vector $\bar{\pi}(x)$ are zero, by construction. We then denote by $\pi(x)$ the vector of $\mathbb{R}^{n}$ such that :

$$
(\pi(x), 0)=\bar{\pi}(x)
$$

Remark 2.1 An easy consequence of the subsituability condition is that the liquidation function $\ell$ is Lipschitz on its effective domain. Therefore,

$$
\pi \text { is Lipschitz continuous and } \pi\left(L_{d}^{\infty}\right) \subset L_{n}^{\infty} .
$$


Example 2.1 (Proportional transaction costs) Let $\lambda=\left(\lambda_{i j}\right)_{1 \leq i, j \leq d}$ be a matrix with nonnegative entries. For all $(i, j)$, the coefficient $\lambda_{i j}$ is the proportional transaction cost for transfers from asset $i$ to asset $j$. This means that transferring an amount $M \geq 0$ from asset $i$ to asset $j$ requires a transaction fee of $\lambda_{i j} M$. A transfer matrix is a $(d \times d)$-matrix with non-negative entries. Each component $(i, j)$ of such a matrix defines the amount transferred from asset $i$ to asset $j$. Given a transfer matrix $a=\left(a_{i j}\right)_{1 \leq i, j \leq d}$, and a portfolio $x \in \mathbb{R}^{d}$, we denote by $x(a)$ the portfolio defined by :

$$
x(a)_{i}:=x_{i}+\sum_{j=1}^{d} a_{j i}-\left(1+\lambda_{i j}\right) a_{i j} \text { for all } i=1, \ldots, d,
$$

i.e. $x(a)$ is the portfolio obtained from $x$ after operating the transfers defined by the transfer matrix $a$. In this financial market, it is natural to define the following relation on the set portfolios :

$$
x \succeq 0 \text { if and only if } x(a) \in \mathbb{R}_{+}^{d} \text { for some transfer matrix } a .
$$

See Kabanov (1999). Clearly, the set $K:=\left\{x \in \mathbb{R}^{d}: x \succeq 0\right\}$ is a closed convex cone, and $\succeq$ is a partial ordering on $\mathbb{R}^{d}$ satisfying the conditions (??) and (??).

\section{$2.3(d, n)$-Coherent risk measures}

We extend the notion of coherent risk measure introduced previously in ADEH to allow for random portfolios valued in $\mathbb{R}^{d}$. Each component of this portfolio corresponds to a specific security market. The motivation is that investors are in general not able to aggregate their portfolio because of liquidity problems and/or transaction costs between the different security markets. - In order for a random portfolio $X$ to be acceptable in terms of "risk", the regulator/supervisor recommends that some deterministic portfolio $\bar{x}$ be added to the position. We then say that $\bar{x}$ cancels the risk induced by $X$ if the aggregate portfolio $X+\bar{x}$ is acceptable by the regulator/supervisor in the sense of the risk measure. The risk measure of the portfolio $X$ will consist of the collection of such deterministic portfolios $\bar{x}$. - The integer $d$, representing the dimensionality of the portfolio $X(\omega)$, is typically large since the firm has positions on many different securities markets. Although regulator/supervisor can possibly recommend any deterministic portfolio $\bar{x} \in \mathbb{R}^{d}$ which cancels the risk of $X$, it is natural to restrict $\bar{x}$ to have a small number $n \leq d$ of non-zero entries. This reduction can be obtained by means of some aggreagation procedure either of the initial random portfolio $X$ or of the deterministic portfolio $\bar{x}$, see Sections ?? and ?? for further discussion of this issue. - For instance, when an amount of cash in Dollars is recommended to be added to the position, we have $n=1$. When the regulator/supervisor recommends to add two different amounts of cash in Dollars and in Euros, we are in the 
situation $n=2$. - By possibly rearranging the components of $\bar{x}$, we shall consider that its last $d-n$ components are zero, for some integer $n \leq d$. This suggests the following notation (which will be used throughout the paper) :

$$
\text { for all } x \in \mathbb{R}^{n}, \quad \bar{x}:=(x, 0) \in \mathbb{R}^{d} .
$$

In conclusion, the notion of $(d, n)$-risk measure should be defined as a map from $L_{d}^{\infty}$ (the set of bounded random portfolios) into the subsets of $\mathbb{R}^{n}$. We suggest the following definition which will be shown to be a convenient extension of ADEH to our context.

Definition 2.1 $A(d, n)$-coherent risk meaure is a set-valued map $R: L_{d}^{\infty} \longrightarrow \mathbb{R}^{n}$ satisfying the following axioms : A0 - For all $X \in L_{d}^{\infty}, R(X)$ is closed, and $0 \in R(0) \neq$ $\mathbb{R}^{n} ; \mathbf{A 1}$ - For all $X \in L_{d}^{\infty}: X \succeq 0 P-$ a.s. $\Longrightarrow R(0) \subset R(X) ; \mathbf{A 2}$ - For all $X, Y \in L_{d}^{\infty}$, $R(X)+R(Y) \subset R(X+Y) ; \mathbf{A} \mathbf{3}$ - For all $t>0$ and $X \in L_{d}^{\infty}, R(t X)=t R(X) ; \mathbf{A} 4$ - For all $x \in \mathbb{R}^{n}$ and $X \in L_{d}^{\infty}, R(X+\bar{x})=\{-x\}+R(X)$.

Remark 2.2 Let us specialize the discussion to the one-dimensional setting $d=n=1$. Starting from a set-valued mapping $R: L_{1}^{\infty} \longrightarrow \mathbb{R}$ satisfying A0, we define

$$
\rho(x):=\min R(x)>-\infty .
$$

Assume that $R(X)$ coincides with $[\rho(X),+\infty)$ (A2 and A3 will guarantee that $R(X)$ is comprehensive which ensures that in the one dimensional case $R$ is of the above form, see Property ?? below). Then, it is easily checked that $R$ satisfies A1-A2-A3-A4 if and only if $\rho$ is a coherent risk measure in the sense of ADEH [?] and Delbaen [?].

Before going any further, we briefly comment Axioms A0 through A4 introduced in the previous definition. - The first requirement in A0 is natural, and only needed for technical reasons. Then, A0 says that 0 is a deterministic portfolio which allows to cancel the risk of the null portfolio. The condition $R(0) \neq \mathbb{R}^{n}$ is assumed to avoid the trivial case $R(X)$ $=\mathbb{R}^{n}$ for all $X \in L_{d}^{\infty}$, see Remark ??. - A1 says that any deterministic portfolio in $R(0)$ allows to cancel the risk of a portfolio $X$, whenever $X \succeq 0$. - A2 is the usual reduction property by risk aggregation : let $x$ (resp. $y$ ) be a deterministic portfolio in $\mathbb{R}^{n}$ which cancels the risk of $X$ (resp. $Y$ ). Then $x+y$ cancels the risk of the aggregate risk $X+Y$. A3 is the usual positive homogeneity property of the risk measure. - A4 is the analogue of the translation invariance axiom introduced in ADEH.

\section{$2.4(d, n)$-acceptance sets}

An alternative way of defining risk measures is provided by the notion of acceptance set, i.e. the set of random portfolios $X \in L_{d}^{\infty}$ which are viewed as free from risk by the supervisor/regulator. 
Definition 2.2 $A(d, n)$-acceptance set is a closed convex cone $\mathcal{A}$ of $L_{d}^{\infty}$, containing $L_{d}^{\infty}(K)$, and such that $\mathbb{R}^{n} \times\{0\}^{d-n} \not \subset \mathcal{A}$.

Remark 2.3 This definition is motivated by the following observation. Let $R$ be a $(d, n)$-coherent risk measure. Then $\mathcal{A}:=\left\{X \in L_{d}^{\infty}: R(0) \subset R(X)\right\}$ is a $(d, n)$-acceptance set in the sense of the above definition. This claim is a direct consequence of the properties stated in the subsequent section.

We now show that the notions of acceptance sets is directly connected to coherent risk measures.

Theorem 2.1 Let $\mathcal{A}$ be a subset of $L_{d}^{\infty}$, and define the set-valued map $R_{\mathcal{A}}: L_{d}^{\infty} \longrightarrow \mathbb{R}^{n}$ by

$$
R_{\mathcal{A}}(X):=\left\{x \in \mathbb{R}^{n}: X+\bar{x} \in \mathcal{A}\right\}
$$

Then, $\mathcal{A}$ is a $(d, n)$-acceptance set if and only if $R_{\mathcal{A}}$ is a $(d, n)$-coherent risk measure.

Proof. (i) Let $\mathcal{A}$ be a $(d, n)$-acceptance set. Clearly, $R_{\mathcal{A}}$ has closed values as $\mathcal{A}$ is closed. Moreover, since $0 \in \mathcal{A}$ and $\mathbb{R}^{n} \times\{0\}^{d-n} \not \subset \mathcal{A}$, we have $0 \in R_{\mathcal{A}}(0) \neq \mathbb{R}^{n}$. Hence $R_{\mathcal{A}}$ satisfies A0. To see that A1 holds, take an arbitrary $x \in R_{\mathcal{A}}$ together with some $X \in L^{\infty}(K)$. Then, by the definition of acceptance sets, both $X$ and $\bar{x}$ are contained in $\mathcal{A}$, and therefore $X+\bar{x}$ $\in \mathcal{A}$, i.e. $x \in R(X)$. That $R_{\mathcal{A}}$ satisfies conditions $\mathrm{A} 2$ and $\mathrm{A} 3$ follows easily from the fact that $\mathcal{A}$ is a convex cone. Finally $\mathrm{A} 4$ is directly obtained from the definition of $R_{\mathcal{A}}$. (ii) Now, suppose that $R_{\mathcal{A}}$ is a $(d, n)$-coherent risk measure. In order to prove that $\mathcal{A}$ is a $(d, n)$-acceptance set, we first observe that

$$
\mathcal{A}=\left\{X \in L_{d}^{\infty}: R_{\mathcal{A}}(0) \subset R_{\mathcal{A}}(X)\right\}
$$

Then, it is easily seen that conditions A0-A1-A2-A3 imply that $\mathcal{A}$ is a convex cone of $L_{d}^{\infty}$ containing $L_{d}^{\infty}(K)$ and not including the whole deterministic space $\mathbb{R}^{n} \times\{0\}^{d-n}$. The closedness of $\mathcal{A}$ in the $L_{d}^{\infty}$ norm follows from Property ?? reported in the subsequent section.

\subsection{Example : the vector $\mathrm{WCE}_{\alpha}$}

In $\mathrm{ADEH}$, the authors propose the use of the worst conditional expectation measure of risk defined by :

For $X \in L^{\infty}: \operatorname{WCE}_{\alpha}(X):=-\inf _{B \in \mathcal{F}^{\alpha}} E[X \mid B]$ where $\mathcal{F}^{\alpha}:=\{B \in \mathcal{F}: P[B]>\alpha\}$, 
and the level $\alpha$ is a given parameter in $(0,1)$. The corresponding acceptance set is given by :

$$
\mathcal{A}_{\mathrm{WCE}_{\alpha}}=\left\{X \in L^{\infty}: E[X \mid B] \geq 0 \text { for all } B \in \mathcal{F}^{\alpha}\right\} .
$$

The functional $W C E_{\alpha}$ is a coherent risk measure, in the sense of ADEH, which appears naturally as a good alternative for the (non-coherent) Value-at-Risk measure. We now provide an extension of this coherent risk measure to our multi-dimensional framework. Let $J$ be a closed convex cone of $\mathbb{R}^{d}$ such that :

$$
K \subset J \quad \text { and } \quad J \neq \mathbb{R}^{d}
$$

and define the subset of $L_{d}^{\infty}$ :

$$
\mathcal{A}_{\alpha}^{J}:=\left\{X \in L_{d}^{\infty}: E[X \mid B] \in J P-\text { a.s. for all } B \in \mathcal{F}^{\alpha}\right\} .
$$

Observe that $\mathcal{A}_{\alpha}^{J}$ coincides with $\mathcal{A}_{\mathrm{WCE}_{\alpha}}$ when $d=1$. Clearly $\mathcal{A}_{\alpha}^{J}$ is a closed convex cone of $L_{d}^{\infty}$ containing $L_{d}^{\infty}(K)$. Also, for all positive integer $n \leq d, \mathcal{A}_{\alpha}^{J}$ does not contain the deterministic set $\mathbb{R}^{n} \times\{0\}^{d-n}$. Hence $\mathcal{A}_{\alpha}^{J}$ is a $(d, n)$-acceptance set, and the set-valued map :

$$
W C E_{\alpha}^{J}(X):=R_{\mathcal{A}_{\alpha}^{J}}(X)=\left\{x \in \mathbb{R}^{n}: X+\bar{x} \in \mathcal{A}_{\alpha}^{J}\right\}
$$

defines a $(d, n)$-coherent risk measure. This is a natural extension of the worst conditional expectation risk measure to the multi-dimensional framework. Notice that the risk measure $W C E_{\alpha}$ is shown to coincide with the Tail $V a R$ in the one-dimensional case, under suitable conditions, and is therefore as easy to compute in practice as the VaR measure. We leave for future research the possible extensions of these results to our multi-dimensional framework.

\section{Properties of coherent risk measures}

We now derive some properties of $(d, n)$-coherent risk measures as defined in Definition ??.

Property 3.1 $R(X)$ is a closed convex subset of $\mathbb{R}^{n}, R(0)$ is a closed convex cone of $\mathbb{R}^{n}$, and

$$
R(X)=R(X)+R(0) \text { for all } X \in L_{d}^{\infty} .
$$

Proof. By A0, $R(X)$ is closed for all $X \in L_{d}^{\infty}$. From A2 and A3, we have

$$
t R(X)+(1-t) R(X) \subset R(t X+(1-t) X)=R(X) \text { for all } t \in[0,1] .
$$

This proves that $R(X)$ is convex. Since $0 \in R(0)$ by $\mathrm{A} 0$, and $t R(0)=R(0)$ by $\mathrm{A} 3$, we deduce that $R(0)$ is a closed convex cone. Finally, using again A2, we see that $R(X)+R(0)$ $\subset R(X)$, and the equality follows from the fact that $0 \in R(0)$. 
Remark 3.1 It follows from Property ?? that A1 can be written in :

$$
\text { A1 }-0 \in R(X) \text { for all } X \in L^{\infty}(K) \text {. }
$$

Similarly, the acceptance set associated with a $(d, n)$-coherent risk measure defined in Remark ?? can also be written in :

$$
\mathcal{A}=\left\{X \in L_{d}^{\infty}: 0 \in R_{\mathcal{A}}(X)\right\}
$$

the next result requires the following additional notations :

$$
K_{n}:=\left\{x \in \mathbb{R}^{n}: \bar{x} \in K\right\} \quad \text { and } \quad \vec{R}_{0}:=R(0) \cap-R(0) .
$$

Observe that $\vec{R}_{0}$ is a vector space.

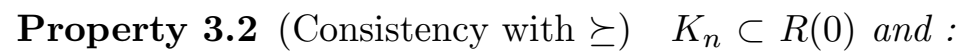

$$
\operatorname{int}\left(-K_{n}\right) \cap R(0)=\left(-K_{n} \backslash \vec{R}_{0}\right) \cap R(0)=\emptyset .
$$

Proof. 1. To see that $K_{n} \subset R(0)$, fix some $x \in K_{n}$. By definition of the partial ordering, we have $\bar{x} \succeq 0$, and therefore $R(0) \subset R(\bar{x})=\{-x\}+R(0)$ by A1 and A4. Hence $x \in R(0)$ and the required inclusion follows from the arbitrariness of $x \in K_{n}$. 2. For $y \in \mathbb{R}^{n}$, set $D_{y}$ $:=\{y\}^{\circ}=\left\{x \in \mathbb{R}^{n}: x y \geq 0\right\}$. Clearly, $R(0)^{\circ}=\left\{y \in \mathbb{R}^{n}: x y \geq 0\right.$ for all $\left.x \in R(0)\right\}=$ $\left\{y \in \mathbb{R}^{d}: R(0) \subset D_{y}\right\}$. Observe that $R(0)^{\circ} \neq\{0\}$ since $R(0)$ is a convex cone by Property ??, and $R(0) \neq \mathbb{R}^{n}$ by A0. From the bipolar theorem, it follows that $R(0)=\left\{x \in \mathbb{R}^{n}\right.$ : $x \in D_{y}$ for all $\left.y \in R(0)^{\circ}\right\}$. This proves in particular that :

$$
x \notin \vec{R}_{0} \quad \text { iff } \quad x \notin \partial D_{y} \text { for some } y \in R(0)^{\circ},
$$

where $\partial D_{y}=D_{y} \cap-D_{y}$. Now take an arbitrary $x \in \operatorname{int}\left(-K_{n}\right)$. Then $x \in \operatorname{int}\left(-D_{y}\right)$ for all $y \in R(0)^{\circ}$, which proves that $x \notin \vec{R}_{0}$ from (??). Hence $\operatorname{int}\left(-K_{n}\right) \subset\left(-K_{n} \backslash \vec{R}_{0}\right)$. 3 . Using again (??) we see that, for all $x \in-K_{n}$ with $x \notin \vec{R}_{0}$, we have $x \in-K_{n} \cap \operatorname{int}\left(-D_{y}\right)$ $\subset-D_{y} \cap \operatorname{int}\left(-D_{y}\right)=\operatorname{int}\left(-D_{y}\right)$ for some $y \in R(0)^{\circ}$. This proves that $x \notin R(0)$ since $R(0) \subset D_{y}$. We have then proved that $\left(-K_{n} \backslash \vec{R}_{0}\right) \cap R(0)=\emptyset$, and the proof is complete.

Remark 3.2 (i) Suppose that $\vec{R}_{0}=\{0\}$. Then Property ?? reduces to $-K_{n} \cap R(0)=\emptyset$. This means that non-positive deterministic portfolios (in the sense of $\succeq$ ) can not cancel the risk of the null portfolio. (ii) Since $K_{n} \subset R(0)$, we have $K_{n} \cap-K_{n} \subset \vec{R}_{0}$. Therefore the above case $\vec{R}_{0}=\{0\}$ implies that there are effective frictions between the first $n$ assets. 
Property 3.3 (Monotonicity) (i) Let $X, Y \in L_{d}^{\infty}$ be such that $X \succeq Y$. Then, $R(Y) \subset$ $R(X)$. (ii) Let $X \in L_{d}^{\infty}$ be such that $\bar{a} \succeq X \succeq \bar{b}$ for some $a, b \in \mathbb{R}^{n}$. Then :

$$
\{-b\}+R(0) \subset R(X) \subset\{-a\}+R(0) .
$$

(iii) For all $X \in L_{d}^{\infty}$, we have $\left\{\|\pi(X)\|_{\infty} \mathbf{1}\right\}+R(0) \subset R(X)$.

Proof. By A1, we have $R(0) \subset R(X-Y)$ whenever $X \succeq Y$. Using A2 together with Property ??, it follows that $R(Y)=R(Y)+R(0) \subset R(Y)+R(X-Y) \subset R(X)$, and the proof of (i) is completed. Claim (ii) is easily obtained from (i) by use of A4. To prove (iii), observe that $X \succeq \bar{\pi}(X) \succeq-\|\pi(X)\|_{\infty} \mathbf{1}$ by definition of $\pi$, and apply again (i).

Remark 3.3 A direct consequence of Property ?? (iii) is that the condition $R(0) \neq \mathbb{R}^{n}$ contained in A0 implies that :

$$
R(X) \neq \mathbb{R}^{n} \text { for all } X \in L_{d}^{\infty}
$$

Remark 3.4 (i) Let $X, Y$ be two random portfolios in $L_{d}^{\infty}$ with $Y \in \vec{K}:=K \cap-K$ a.s. Then, $X+Y \succeq X$ and $X \succeq X+Y$ a.s. From Property ?? (i), it follows that

$$
R(X+Y)=R(X) .
$$

(ii) Let $\operatorname{pr}_{\vec{K}}$ denote the orthogonal projection on the vector space $\vec{K}$, and set $\tilde{X}:=$ $X-\operatorname{pr}_{\vec{K}}(X)$. Part (i) of this remark provides an obvious extension of the vector valued risk measure $R$ to the space $L_{d}^{\infty}+L_{d}^{0}(\vec{K})$ by setting :

$$
R(X)=R(\tilde{X}) \text { for all } X \in L_{d}^{\infty}+L_{d}^{0}(\vec{K}) .
$$

Remark 3.5 It is easily checked that the ordering defined on the portfolios is complete if and only if $K$, i.e. $K=\left\{x \in \mathbb{R}^{d} \mid a x \geq 0\right\}$ for some vector $a \in \mathbb{R}_{+}^{d} \backslash\{0\}$. By possibly multiplying $a$ by a constant, we can find an integer $i \in\{1, \ldots, d\}$ satisfying $a_{i}=1$. Then, for any portfolio $X \in L_{d}^{\infty}$, one has $X \succeq(a X) 1^{i} \succeq X$. From the monotonicity Property ??, it follows that :

$$
R(X)=R\left(a X \mathbf{1}^{i}\right)
$$

and we are essentially back to the one-dimensional case of ADEH. 
Remark 3.6 By the same argument as in Property ?? (iii), we have :

$$
\left\{\|\pi(X)\|_{\infty} \mathbf{1}\right\}+R(0) \subset R(X) \subset\left\{-\|\pi(-X)\|_{\infty} \mathbf{1}\right\}+R(0) \text { for all } X \in L_{d}^{\infty} .
$$

It is an easy exercise to show that this property implies that $R(0)$ is the asymptotic cone of $R(X)$, i.e.

$$
R(0)=\bigcap_{k \geq 0} \operatorname{cone}\{x \in R(X):|x| \geq k\} .
$$

Property 3.4 (Self-consistency) For all $X \in L_{d}^{\infty}$,

$$
\begin{aligned}
R(X) & =\left\{x \in \mathbb{R}^{n}: 0 \subset R(X+\bar{x})\right\} \\
& =\left\{x \in \mathbb{R}^{n}: R(0) \subset R(X+\bar{x})\right\} .
\end{aligned}
$$

Proof. The second equality is a direct consequence of Property ??. To see that the first equality holds, it suffices to observe that $x \in R(X)$ if and only if $0 \in\{-x\}+R(X)=$ $R(X+\bar{x})$ by $\mathrm{A} 4$.

$\square$ The final property of this

section states the continuity of the set-valued map $R$. We recall that - a set valued map $F$ from a metric vector space $U$ into a metric vector space $V$ is said to be continuous if it is both lower-semicontinuous and upper-semicontinuous, $-F$ is lower semicontinuous at some $u \in U$ if for all $v \in F(u)$ and for any sequence $\left(u^{n}\right)_{n} \subset \operatorname{dom}(F)$ converging to $u$, there is a sequence $v^{n} \in F\left(u^{n}\right)$ such that $v^{n} \longrightarrow v,-F$ is upper-semicontinuous at some $u \in U$ if for all $\varepsilon>0$, there exists a constant $\eta>0$ such that $F\left(u+\eta B_{U}\right) \subset F(u)+\varepsilon B_{V}$; here, $B_{U}$ and $B_{V}$ are the unit balls of $U$ and $V$.

Property 3.5 (Continuity) (i) For all $X, Y \in L_{d}^{\infty}$,

$$
R(Y)+\left\{\|\pi(Y-X)\|_{\infty} \mathbf{1}\right\} \quad \subset R(X) \subset R(Y)-\left\{\|\pi(X-Y)\|_{\infty} \mathbf{1}\right\} .
$$

(ii) The set-valued map $R$ is continuous on $L_{d}^{\infty}$.

Proof. We first prove (i). By A2 together with Properties ?? (iii) and ??, we see that

$$
\begin{aligned}
R(X) \subset R(X-Y)+R(Y) & \subset-\left\{\|\pi(X-Y)\|_{\infty} \mathbf{1}\right\}+R(0)+R(Y) \\
& =-\left\{\|\pi(X-Y)\|_{\infty} \mathbf{1}\right\}+R(Y) .
\end{aligned}
$$

By symmetry, we also have $R(Y) \subset-\left\{\|\pi(Y-X)\|_{\infty} \mathbf{1}\right\}+R(X)$, and the proof of (i) is complete. (ii) To see that $R$ is lower-semicontinuous at some $X \in L_{d}^{\infty}$, take some $y \in R(X)$ 
together with an $L_{d}^{\infty}$ sequence $\left(X^{k}\right)_{k}$ converging to $X$ in the $L_{d}^{\infty}$ norm. From the right left-side inclusion of (i), we deduce the existence of a sequence $y^{k} \in R\left(X^{k}\right)$ such that

$$
y=y^{k}-\left\|\pi\left(X-X^{k}\right)\right\|_{\infty} \mathbf{1} .
$$

Since $\pi$ is a Lipschitz-continuous map on its domain, see Remark ??, we see that $\pi\left(X-X^{k}\right)$ $\longrightarrow 0$ in $L_{d}^{\infty}$, and therefore $y^{k} \longrightarrow y$. It remains to prove that $R$ is upper-semicontinuous. Let $B$ denote the unit ball of $L_{d}^{\infty}$, and take an arbitrary $\varepsilon>0$. By Lipschitz-continuity of $\pi$ on its domain, one can find some $\eta>0$ such that $\pi(X-Y) \in \varepsilon B$ for all $Y \in X+\eta B$. We now use the right hand-side inclusion of (i) to see that $R(Y) \subset R(X)-\{\pi(X-Y) \mathbf{1}\}$ $\subset R(X)+\varepsilon B$ for all $Y \in X+\eta B$.

\section{Dual representation of $(d, n)$-coherent risk measures}

In this section, we provide a dual representation of coherent risk measures. In the onedimensional case, this result was shown to have important practical implications, since it proves the universality of the scenario-based methods for providing coherent measures of risk in the sense of Definition ??. The SPAN method is an example of such scenario-based measure of risk. The proof was first established in ADEH for finite $\Omega$, and further extended to a general probability space by Delbaen [?]. We first need to introduce some additional notations. The dual space of $L_{d}^{\infty}$, denoted by $b a_{d}$, is the set of bounded additive set functions $\mu$ on $(\Omega, \mathcal{F})$ with the property that $\mathbb{P}(A)=0$ implies $\mu(A)=0$. The space $b a_{d}$ is endowed with the norm of total variation, i.e. $\|\mu\|=\sup \left\{\sum_{i=1}^{k}\left|\mu\left(A_{i}\right)\right|: A_{1}, \ldots, A_{k}\right.$ disjoint subsets of $\mathcal{F}\}$, for all $\mu \in b a_{d}$. We shall write $E_{\mu}[X]$ for the duality mapping between a random variable $X \in L_{d}^{\infty}$ and a measure $\mu \in b a_{d}$. The countably additive elements of $b a_{d}$ can be identified with $L_{d}^{1}$ random variables, and are more interesting from the economic viewpoint as they can be interpreted as pricing kernels. The strictly positive (in some sense) elements of $L_{d}^{1}$ are of particular interest in finance since they can be viewed as pricing kernels consistent with the notion of no-arbitrage, see Theorems ?? and ?? below. Finally, we shall denote by $L_{d}^{\infty}(K)$ the subset of $L_{d}^{\infty}$ consisting of all $K$-valued random variables, i.e. non-negative random variables in the sense of $\succeq$. The positive orthant of $b a_{d}$ is defined accordingly by :

$$
b a_{d}(K):=\left\{\mu \in b a_{d}: E_{\mu}[X] \geq 0 \text { for all } X \in L_{d}^{\infty}(K)\right\} .
$$

Theorem 4.1 Let $R: L_{d}^{\infty} \longrightarrow \mathbb{R}^{n}$ be a set valued map. The following claims are equivalent. (1) $R$ is a $(d, n)$-coherent risk measure. (2) There exists a non-zero convex $\sigma\left(b a_{d}, L_{d}^{\infty}\right)$-closed cone, $\mathcal{P}_{b a}$ of $b a_{d}(K)$ such that for all $X \in L_{d}^{\infty}$ :

$$
R(X)=\left\{x \in \mathbb{R}^{n}: \inf _{\mu \in \mathcal{P}_{b a}} E_{\mu}(X+\bar{x}) \geq 0\right\} .
$$


Proof. The implication $(2) \Longrightarrow(1)$ follows by direct verification of Axioms A1-A2-A3A4. We then concentrate on $(1) \Longrightarrow(2)$. We adapt the argument in Delbaen [?] to our set-valued framework. Consider the subset of $L_{d}^{\infty}$

$$
C:=\left\{X \in L_{d}^{\infty}: 0 \in R(X)\right\},
$$

together with its positive polar cone

$$
C^{\circ}:=\left\{\mu \in b a_{d}: E_{\mu}[X] \geq 0 \text { for all } X \in C\right\} .
$$

First, observe that $C$ contains the positive orthant $L_{d}^{\infty}(K)$ and $C \neq \mathbb{R}^{n}$. Therefore $\{0\} \neq$ $C^{\circ} \subset b a_{d}(K)$. Also, by definition of the duality mapping, $C^{\circ}$ is $\sigma\left(b a_{d}, L_{d}^{\infty}\right)$-closed. Next, $C$ is clearly a convex cone of $L_{d}^{\infty}$. It is easily checked that it is closed in the sense of the $L_{d}^{\infty}$ norm : let $\left(X^{k}\right)_{k}$ be a sequence in $C$ converging to some $X \in L_{d}^{\infty}$, then $R(0) \subset R\left(X^{k}\right)$ for all $k \in \mathbb{N}$, and therefore $R(0) \subset \lim \sup _{k} R\left(X^{k}\right)=R(X)$ by the continuity Property ?? (ii). We are then in the context of the bipolar Theorem which provides :

$$
C=\left\{X \in L_{d}^{\infty}: \inf _{\mu \in C^{\circ}} E_{\mu}[X] \geq 0\right\} .
$$

Finally, it follows from the self-consistency Property ?? that :

$$
\begin{aligned}
R(X) & =\left\{x \in \mathbb{R}^{n}: 0 \in R(X+\bar{x})\right\} \\
& =\left\{x \in \mathbb{R}^{n}: X+\bar{x} \in C\right\} \\
& =\left\{x \in \mathbb{R}^{n}: \inf _{\mu \in C^{\circ}} E_{\mu}[X+\bar{x}] \geq 0\right\},
\end{aligned}
$$

and the statement of the theorem holds with $\mathcal{P}_{b a}=C^{\circ}$. $\quad \square$ We next follow Delbaen [?] to see under which circumstances the minimization in the dual representation of Theorem ?? can be restricted to a subset of $L_{d}^{1}$.

Definition 4.1 $A(d, n)$-coherent risk measure $R: L_{d}^{\infty} \longrightarrow \mathbb{R}^{n}$ is said to satisfy the Fatou property if, for all $X \in L_{d}^{\infty}$,

$$
\liminf _{k \rightarrow \infty} R\left(X^{k}\right) \subset R(X),
$$

for any bounded sequence $\left(X^{k}\right)_{k \in \mathbb{N}}$ of $L_{d}^{\infty}$ converging to $X$ in probability.

Theorem 4.2 Let $R: L_{d}^{\infty} \longrightarrow \mathbb{R}^{n}$ be a $(d, n)$-coherent risk measure. The following properties are equivalent (1) There exists a closed subset $\mathcal{P}_{\sigma}$ of $L_{d}^{1}\left(K^{\circ}\right)$ such that for all $X \in L_{d}^{\infty}$,

$$
R(X)=\left\{x \in \mathbb{R}^{n}: \inf _{\mu \in \mathcal{P}_{\sigma}} E_{\mu}(X+\bar{x}) \geq 0\right\} .
$$

(2) The convex cone $C=\left\{X \in L_{d}^{\infty}: R(0) \subset R(X)\right\}$ is $\sigma\left(L_{d}^{\infty}, L_{d}^{1}\right)$-closed. (3) The $(d, n)$-coherent risk measure $R$ satisfies the Fatou property. 
Proof. We again adapt the argument of Delbaen [?] to the set-valued framework. (1) $\Longrightarrow$ (3) Let $\left(X^{k}\right)_{k \in \mathbb{N}}, X \in L_{d}^{\infty}$ such that the sequence $\left(X^{k}\right)_{k \in \mathbb{N}}$ is bounded and converges to $X$ in probability. Let $x$ be an arbitrary element of $\liminf _{k} R\left(X^{k}\right)$. Then, there exists a sequence $\left(x^{k}\right)_{k \in \mathbb{N}}$ in $\mathbb{R}^{n}$ such that $x^{k} \in R\left(X^{k}\right)$ for all $k \in \mathbb{N}$, and $x^{k} \longrightarrow x$. Then, for all $\mu \in \mathcal{P}_{\sigma}$,

$$
E_{\mu}\left(X^{k}+\bar{x}^{k}\right) \geq 0 \text { for all } \mu \in \mathcal{P}_{\sigma} \text { and } k \in \mathbb{N},
$$

and

$$
E_{\mu}(X+\bar{x}) \geq \limsup _{k \rightarrow \infty} E_{\mu}\left(X^{k}+\bar{x}^{k}\right) \geq 0 \text { for all } \mu \in \mathcal{P}_{\sigma}
$$

implying that $x \in R(X)$. (3) $\Longrightarrow(2)$ From Grothendieck [?] (supp. ex. 1 p204), and since $C$ is a cone, it is sufficient to check that $C \cap B$ is closed in probability ( $B$ is the closed unit ball of $\left.L_{d}^{\infty}\right)$. Let $\left(X^{k}\right)$ be a sequence of $C$ such that $\left\|X^{k}\right\|_{\infty} \leq 1$ for all $k \in \mathbb{N}$ and converging in probability to some $X \in L_{d}^{\infty}$. From the Fatou property, one has $\liminf _{k} R\left(X^{k}\right) \subset R(X)$ and since $R(0) \subset R\left(X^{k}\right)$ for all $k \in \mathbb{N}$, one obtains that $R(0) \subset \liminf _{k} R\left(X^{k}\right) \subset R(X)$ and thus $X \in C$. (2) $\Longrightarrow$ (1) Follows the lines of the proof of Theorem ??, and take the polar of $C$ in $L_{d}^{1}$. For completeness, we report a direct proof of $(2) \Longrightarrow(3)$. Assume that $C$ is $\sigma\left(L_{d}^{\infty}, \mathrm{L}_{d}^{1}\right)$-closed. Let $\left(X^{k}\right)_{k \in \mathbb{N}}, X \in L_{d}^{\infty}$ such that the sequence $\left(X^{k}\right)_{k \in \mathbb{N}}$ is bounded and converges to $X$ in probability. Observe that $X^{k} \longrightarrow X$ weakly in $\sigma\left(L_{d}^{\infty}, \mathrm{L}_{d}^{1}\right)$, since the sequence $\left(X^{k} Y\right)_{k}$ is uniformly integrable for all $Y \in \mathrm{L}_{d}^{1}$. Let $x \in \liminf _{k} R\left(X^{k}\right)$, then, there exists a sequence $\left(x^{k}\right)_{k \in \mathbb{N}}$ in $\mathbb{R}^{n}$ such that for all $k \in \mathbb{N}, x^{k} \in R\left(X^{k}\right)$ and $x^{k} \longrightarrow$ $x$. From the self-consistency Property ??, $R(0) \subset R\left(X^{k}+\bar{x}^{k}\right)$ for all $k \in \mathbb{N}$, meaning that $X^{k}+\bar{x}^{k} \in C$. Since $C$ is $\sigma\left(L_{d}^{\infty}, \mathrm{L}_{d}^{1}\right)$-closed, one obtains also that $X+\bar{x} \in C$. Using again the self-consistency Property ??, we see that $R(0) \subset R(X+\bar{x})=\{-x\}+R(X)$. Since $0 \in R(0)$, this shows that $x \in R(X)$.

\section{Relevance of a $(d, n)$-coherent risk measure}

In this section, we focus on the notion of relevancy of a coherent risk measure, which was introduced by Delbaen [?] in the one-dimensional case. In Theorem ??, a coherent risk measure is represented in terms of a family of state price densities $\mu$ valued in $K^{\circ}$. A natural question, which is closely related to the notion of no-arbitrage, is whether such state price densities are strictly positive in some sense.

Remark 5.1 Recall that the closed convex cone $K$ contains the positive orthant $\mathbb{R}_{+}^{d}$ of $\mathbb{R}^{d}$. Then $K^{\circ} \subset \mathbb{R}_{+}^{d}$. A first notion of strict positivity of the state price density $\mu$ is the 
requirement that all components $\mu_{i}$ are positive a.s. The following interesting observation follows from the substitutability condition (??) :

$$
\text { for all } \begin{aligned}
y \in K^{\circ}: y_{1}=0 & \Longleftrightarrow y_{i}=0 \text { for all } i=n+1, \ldots, d \\
& \Longleftrightarrow y_{i}=0 \text { for some } i=n+1, \ldots, d .
\end{aligned}
$$

Following Kabanov and Stricker [?], the multi-dimensional framework suggests at least the following definitions of relevancy. Recall the notation $\vec{K}:=K \cap-K$.

Definition 5.1 $A(d, n)$-coherent risk measure $R$ is said to be $\bullet$ weakly relevant if for all $X \in L_{d}^{\infty}(K), 0 \in R(-X) \Longrightarrow P[X \in \operatorname{int}(K)]=0$, • strongly relevant if for all $X \in L_{d}^{\infty}(K)$, $0 \in R(-X) \Longrightarrow P[X \in(K \backslash \vec{K})]=0$.

Observe that any strongly relevant $(d, n)$-coherent risk measure $R$ is weakly relevant. We also have the following equivalent definitions of relevancy.

Proposition 5.1 Let $R$ be a $(d, n)$-coherent risk measure. $\bullet R$ is weakly relevant if and only :

$$
\text { for all } A \in \mathcal{F} \text { and } z \in \operatorname{int}(K), \quad 0 \in R\left(-z \mathbf{1}_{A}\right) \quad \Longrightarrow \quad P(A)=0 \text {. }
$$

- $R$ is strongly relevant if and only if :

$$
\text { for all } A \in \mathcal{F} \text { and } z \in(K \backslash \vec{K}), \quad 0 \in R\left(-z \mathbf{1}_{A}\right) \quad \Longrightarrow P(A)=0 \text {. }
$$

Proof. We only report the proof of the equivalent definition of weak relevancy. Suppose that Condition ?? holds, and let $X \in L_{d}^{\infty}(K)$ be such that $0 \in R(-X)$ and $P[(X \in \operatorname{int}(K)]$ $>0$. Then, there exists $z \in \operatorname{int}(K)$ such that $P[X \succeq z]>0$. Set $A:=\{X \succeq z\}$. Clearly $X \succeq z \mathbf{1}_{A}$ and thus $0 \in R(-X) \in R\left(-z \mathbf{1}_{A}\right)$ by the monotonicity Property ??. This is in contradiction with (??).

Theorem 5.1 Let $R$ be a $(d, n)$-coherent risk measure satisfying the Fatou property, $\mathcal{P}_{\sigma}$ the associated dual set introduced in Theorem ??, and set

$$
\mathcal{P}_{\sigma}^{w}:=\left\{\mu \in \mathcal{P}_{\sigma}: \mu \in K^{\circ} \backslash\{0\} \mathbb{P}-\text { a.s. }\right\}
$$

Then the following claims are equivalent. (1) $R$ is weakly relevant. (2) The set $\mathcal{P}_{\sigma}^{w}$ is nonempty. (3) The set $\mathcal{P}_{\sigma}^{w}$ is dense in $\mathcal{P}_{\sigma}$ in the sense of the $L_{d}^{1}$ norm. (4) There is a set $\mathcal{P} \subset$ $\mathcal{P}_{\sigma}^{w}$ such that:

$$
R(X)=\left\{x \in \mathbb{R}^{n}: \inf _{\mu \in \mathcal{P}} E_{\mu}(X+\bar{x}) \geq 0\right\} .
$$


Proof. We only prove $(1) \Longrightarrow(2)$ as the remaining implications are trivial. Step 1. We first fix an arbitrary event set $A \in \mathcal{F}$ with $P(A)>0$. From the dual representation of $R$ in Theorem ??, we have that

$$
\inf _{\mu \in \mathcal{P}_{\sigma}} E_{\mu}\left[-z \mathbf{1}_{A}\right]<0 \text { for all } z \in \operatorname{int}(K) .
$$

This proves that the set $\zeta_{A}(\mu):=\left\{z \in \operatorname{int}(K): E_{\mu}\left[-z \mathbf{1}_{A}\right]<0\right\}$ is non-empty for some $\mu$ $\in \mathcal{P}_{\sigma}$. We then consider the family of subsets of $\operatorname{int}(K)$ defined by :

$$
\mathcal{Z}_{A}:=\left\{\zeta_{A}(\mu): \mu \in \mathcal{P}_{\sigma}\right\}
$$

The family $\mathcal{Z}_{A}$ has a maximal element $\zeta_{A}\left(\mu_{A}\right)$, for some $\mu_{A} \in \mathcal{P}_{\sigma}$, as it easily checked that it is stable by countable union. We now use an exhaustion argument to see that

$$
\zeta_{A}\left(\mu_{A}\right)=\operatorname{int}(K) \text {, i.e. } E_{\mu_{A}}\left[-z \mathbf{1}_{A}\right]<0 \text { for all } z \in \operatorname{int}(K) \text {. }
$$

Indeed, let $y$ be an arbitrary element in $\operatorname{int}(K) \backslash \zeta_{A}\left(\mu_{A}\right)$. By (??), we deduce the existence of some $\mu \in \mathcal{P}_{\sigma}$ such that $E_{\mu}\left[-y \mathbf{1}_{A}\right]<0$, and we observe that $\zeta_{A}\left(\mu_{A}\right) \cup\{y\} \subset \zeta_{A}\left(\mu_{A}+\mu\right)$, contradicting the maximality of $\mu_{A}$. Step 2. We now want to select some $\hat{\mu} \in \mathcal{P}_{\sigma}$ such that

$$
\zeta_{A}(\hat{\mu})=\operatorname{int}(K) \text { for all } A \in \mathcal{F} \text {. }
$$

With $\mathcal{B}_{+}:=\{B \in \mathcal{B}: P[B]>0\}$ for $\mathcal{B} \subset \mathcal{F}$, we introduce the family

$$
\mathcal{G}:=\left\{\mathcal{B} \subset \mathcal{F}: \bigcap_{B \in \mathcal{B}_{+}} \zeta_{B}(\mu)=\operatorname{int}(K) \text { for some } \mu \in \mathcal{P}_{\sigma}\right\} .
$$

It is again easily checked that $\mathcal{G}$ is stable by countable union. Therefore, it admits a maximal element $\hat{\mathcal{B}}$ associated with some $\hat{\mu} \in \mathcal{P}_{\sigma}$. We now use a second exhaustion argument to see that $\hat{\mathcal{B}}=\mathcal{F}$ which provides (??). Indeed, Let $A$ be an arbitrary element in $\mathcal{F} \backslash \hat{\mathcal{B}}$ with $P[A]>0$. Considering the measure $\mu_{A}$ of Step 1 , it follows that $\zeta_{B}\left(\hat{\mu}+\mu_{A}\right)=\operatorname{int}(K)$ for all $B \in \hat{\mathcal{B}} \cup\{A\}$. This is in contradiction with the maximality of $\hat{\mathcal{B}}$. Step 3. In Step 2, we have proved the existence of some $\hat{\mu} \in \mathcal{P}_{\sigma}$ satisfying (??), or equivalently,

$$
E_{\hat{\mu}}\left(-z \mathbf{1}_{A}\right)<0 \text { for all } z \in \operatorname{int}(K) \text { and } A \in \mathcal{F}_{+} .
$$

This proves that

$$
\hat{\mu} \in \hat{K}:=\left\{y \in \mathbb{R}^{d}: y z>0 \text { for all } z \in \operatorname{int}(K)\right\} \quad P \text { - a.s. }
$$

In order to conclude the proof, it remains to check that $\hat{K}=K^{\circ} \backslash\{0\}$. We only concentrate on the proof of $K^{\circ} \backslash\{0\} \subset \hat{K}$ as the reverse inclusion is trivial. Let $y$ be an arbitrary element in $K^{\circ} \backslash\{0\}$, and assume that $y z=0$ for some $z \in \operatorname{int}(K)$. Let $\varepsilon>0$ be such that 
$(z-\varepsilon y) \in K$. Then, $(z-\varepsilon y) y<0$, contradicting the fact that $y \in K^{\circ}$.

We also have a similar characterization of strongly relevant coherent risk measures which satisfy the Fatou property.

Theorem 5.2 Let $R$ be a $(d, n)$-coherent risk measure satisfying the Fatou property, $\mathcal{P}_{\sigma}$ the associated dual set introduced in Theorem ??, and set

$$
\mathcal{P}_{\sigma}^{s}:=\left\{\mu \in \mathcal{P}_{\sigma}: \mu \in \operatorname{ri}\left(K^{\circ}\right) P-\text { a.s. }\right\}
$$

Then the following claims are equivalent: (1) $R$ is strongly relevant. (2) The set $\mathcal{P}_{\sigma}^{s}$ is non-empty. (3) The set $\mathcal{P}_{\sigma}^{s}$ is dense in $\mathcal{P}_{\sigma}$ in the sense of the $L_{d}^{1}$ norm. (4) There is a set $\mathcal{P} \subset \mathcal{P}_{\sigma}^{s}$ such that :

$$
R(X)=\left\{x \in \mathbb{R}^{n}: \inf _{\mu \in \mathcal{P}} E_{\mu}(X+\bar{x}) \geq 0\right\}
$$

Proof. Following the lines of Steps 1 and 2 in the previous proof, we obtain the existence of some $\hat{\mu} \in \mathcal{P}_{\sigma}$ such that

$$
\hat{\mu} \in \tilde{K}:=\left\{y \in \mathbb{R}^{d}: y z>0 \text { for all } z \in(K \backslash \vec{K})\right\} \quad P-\text { a.s. }
$$

In order to conclude the proof, it remains to check that $\tilde{K}=\operatorname{ri}\left(K^{\circ}\right)$. To se this, we use the following characterization

$$
\operatorname{ri}\left(K^{\circ}\right)=\left\{y \in \mathbb{R}^{d}: x y<\delta(x) \text { for all } x \text { satisfying }-\delta(-x) \neq \delta(x)\right\},
$$

where $\delta(x):=\sup _{y \in K^{\circ}}(x y)$ is the support function of $K^{\circ}$, see Rockafellar [?] Theorem 13.1. Since $K^{\circ}$ is a closed convex cone, $\delta(x)=0$ if $-x \in\left(K^{\circ}\right)^{\circ}=K$, and $+\infty$ otherwise. Then, one can write the above characterization in :

$$
\operatorname{ri}\left(K^{\circ}\right)=\left\{y \in \mathbb{R}^{d}: x y<0 \text { for all } x \in-K \text { with }-x \notin-K\right\}=\tilde{K}
$$

\section{Coherent aggregation of random portfolios}

Definition 6.1 Let $R$ be a $(n, n)$-coherent risk measure. A function $f: \mathbb{R}^{d} \longrightarrow \mathbb{R}^{n}$ is an $R$-coherent portfolio aggregator if PA1 $f(K) \subset R(0)$; PA2 For all $x, y \in \mathbb{R}^{d}$ : $f(x+y)-f(x)-f(y) \in R(0)$; PA3 For all $x \in \mathbb{R}^{d}$ and $t>0: f(t x)-t f(x) \in \vec{R}_{0} ; \mathbf{P A 4}$ For all $x \in \mathbb{R}^{n}$ and $y \in \mathbb{R}^{d}: f(\bar{x}+y)-f(y)-x \in \vec{R}_{0}$. 
We shall discuss some examples of $R$-coherent portfolio aggregators at the end of this section. Our next result requires to introduce a stronger version of $\mathrm{A} 1: \mathbf{A} \mathbf{1}_{s} \quad$ For all $X \in$ $L_{d}^{\infty}$, we have $: X \in R(0) P$-a.s. $\Longrightarrow 0 \in R(X)$.

Remark 6.1 Condition $\mathrm{A} 1_{s}$ is satisfied by deterministic portfolios $x \in \mathbb{R}^{d}$. This is an easy consequence of the self-consistency Property ??.

Remark 6.2 In the case $n=d=1$, conditions $\mathrm{A} 1_{s}$ and $\mathrm{A} 1$ are identical since $R(0)=K$ $=\mathbb{R}_{+}$.

Example 6.1 (Coherent risk measure which does not satisfy $\mathrm{A} 1_{s}$ ) Let $K=\mathbb{R}_{+}^{2}$ be the non-negative orthant of $\mathbb{R}^{2}, \Omega=\left\{\omega_{1}, \omega_{2}\right\}$, and

$$
\mathcal{P}_{\sigma}=\left\{\mu=\left(\mu_{1}, \mu_{2}\right)\right\} \quad \text { where } \mu\left(\omega_{1}\right)=\left(\begin{array}{c}
0.5 \\
0.25
\end{array}\right) \text { and } \mu\left(\omega_{2}\right)=\left(\begin{array}{c}
0.5 \\
0.75
\end{array}\right) .
$$

Clearly, since $K$ is the non-negative orthant of $\mathbb{R}^{2}, \mu \in \mathrm{ba}_{2}(K)$. Therefore, it follows from Theorem ?? that the set valued function :

$$
R(X):=\left\{\left(x_{1}, x_{2}\right) \in \mathbb{R}^{2}: E_{\mu}[X+x]=\sum_{i, j=1}^{2} \mu_{j}\left(\omega_{i}\right)\left(X_{j}\left(\omega_{i}\right)+x_{j}\right) \geq 0\right\}
$$

defines a $(2,2)$ - coherent coherent risk measure. Observe that $R(0)$ is the half space $\left\{x_{1}+\right.$ $\left.x_{2} \geq 0\right\}$. Now consider the random portfolio

$$
X\left(\omega_{1}\right)=(-1,1) \text { and } X\left(\omega_{2}\right)=(0,0),
$$

so that $X\left(\omega_{i}\right) \in R(0)$ for $i=1,2$. But

$$
\sum_{i, j=1}^{2} \mu_{j}\left(\omega_{i}\right) X_{j}\left(\omega_{i}\right)=-0.25
$$

which proves that $0 \notin R(X)$.

The main result of this section shows that $R$-coherent portfolio aggregators $f$ define $(d, n)$-coherent risk measures $\tilde{R}:=R \circ f$ under the additional condition $\mathrm{A} 1_{s}$. We first need to check that $\tilde{R}$ is well-defined. We shall use the notation $\operatorname{pr}_{\vec{R}_{0}^{\perp}}$ for the orthogonal projection on the vector space $\vec{R}_{0}^{\perp}$.

Lemma 6.1 Let $R$ be an $(n, n)$-coherent risk measure, and $f$ an $R$-coherent portfolio aggregator. Then: (i) $f\left(L_{d}^{\infty}\right) \subset L_{d}^{\infty}+L_{d}^{0}\left(\vec{R}_{0}\right)$. (ii) Assume that $R$ satisfies the additional condition $A 1_{s}$. Then $\tilde{R}:=R \circ f$ is well-defined on $L_{d}^{\infty}$. 
Proof. (i) We shall prove that $f$ has the following decomposition :

$$
f=g+h \text { where } g\left(L_{d}^{\infty}\right) \subset L_{d}^{\infty} \text { and } h\left(L_{d}^{\infty}\right) \subset L_{d}^{0}\left(\vec{R}_{0}\right) .
$$

1. Let $y$ be an arbitrary element of the negative polar cone $-R(0)^{\circ}:=\left\{y \in \mathbb{R}^{n}: x y \leq 0\right.$ for all $x \in R(0)\}$. Then, it follows from PA2 and PA3 that the real-valued function $f_{y}$, defined by $f_{y}(x):=y f(x)$, is sublinear. Then, it is locally bounded as a convex function with effective domain $\mathbb{R}^{d}$. By the same argument, we also have that $f_{y}$ is locally bounded for all $y \in R(0)^{\circ}$. Hence,

$$
f_{y}\left(L_{d}^{\infty}\right) \subset L_{d}^{\infty} \text { for all } y \in R(0)^{\circ}+\left(-R(0)^{\circ}\right)
$$

2. Clearly, we have that

$$
\mathbb{R}_{0} \subset\left[R(0)^{\circ}+\left(-R(0)^{\circ}\right)\right]^{\circ}
$$

From the fact that $R(0)^{\circ}$ is a convex cone, it follows that the set $R(0)^{\circ}+\left(-R(0)^{\circ}\right)$ is a vector space, and $\left[R(0)^{\circ}+\left(-R(0)^{\circ}\right)\right]^{\circ}=\left[R(0)^{\circ}+\left(-R(0)^{\circ}\right)\right]^{\perp}$. Since $\vec{R}_{0}$ is also a vector space, we can rewrite (??) equivalently in

$$
R(0)^{\circ}+\left(-R(0)^{\circ}\right) \subset \mathbb{R}_{0}^{\perp} .
$$

In view of (??), this proves that

$$
f_{y}\left(L_{d}^{\infty}\right) \subset L_{d}^{\infty} \text { for all } y \in \vec{R}_{0}^{\perp}
$$

3. Now define

$$
g:=\operatorname{proj}_{\vec{R}_{0}^{\perp}}(f) \text { so that } h:=f-g \text { is valued in } \vec{R}_{0} .
$$

In order to complete the proof of (i), we need to show that $g\left(L_{d}^{\infty}\right) \subset L_{d}^{\infty}$. Suppose to the contrary that

$$
\left|g\left(x^{n}\right)\right| \longrightarrow \infty \text { for some sequence } x_{n} \longrightarrow x \in \mathbb{R}^{d}
$$

and let us work towards a contradiction. Since $\vec{R}_{0}^{\perp}$ is finite dimensional vector space,

$$
y g\left(x_{n}\right) \longrightarrow \infty \text { for some } y \in \vec{R}_{0}^{\perp} \text {. }
$$

We now observe that $y g\left(x^{n}\right)=y f\left(x^{n}\right)-y h\left(x^{n}\right)=y f\left(x^{n}\right)$, so that (??) is in contradiction with (??). (ii) The remaining claim follows by an obvious extension of $R$ to the space $L_{d}^{\infty}+$ $L_{d}^{0}\left(\vec{R}_{0}\right)$. This is obtained exactly by the same argument than in Remark ??, substituting $R(0)$ to $K$. This is possible thanks to $\mathrm{A} 1_{s}$. 
Theorem 6.1 Let $R$ be a $(n, n)$-coherent risk measure, and let $f$ be a mapping from $\mathbb{R}^{d}$ into $\mathbb{R}^{n}$. (i) Suppose that the set-valued map $R \circ f: L_{d}^{\infty} \longrightarrow \mathbb{R}^{n}$ is a $(d, n)$-coherent risk measure. Then $f$ is an $R$-coherent portfolio aggregator. (ii) Conversely, assume that $\mathrm{A}_{1}$ holds, and let $f$ be an $R$-coherent portfolio aggregator. Then the set-valued map $R \circ f$ : $L_{d}^{\infty} \longrightarrow \mathbb{R}^{n}$ is a $(d, n)$-coherent risk measure.

Proof. Set $\tilde{R}:=R \circ f$. 1. Suppose that $\tilde{R}$ is a $(d, n)$-coherent risk measure, and let us

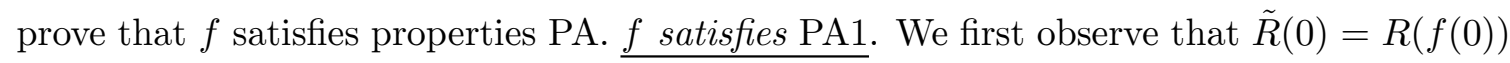
$=\{-f(0)\}+R(0)$. Since $0 \in \tilde{R}(0)$, this proves that $f(0) \in R(0)$. By the self-consistency Property ??, this proves that:

$$
R(0) \subset R(f(0))=\tilde{R}(0)
$$

Now take some $x \in K$ and let us prove that $f(x) \in R(0)$, it follows from A1 that $\tilde{R}(0) \subset$ $\tilde{R}(x)=R(f(x))=\{-f(x)\}+R(0)$. In view of (??), this proves that $R(0) \subset\{-f(x)\}+R(0)$. Since $0 \in R(0)$, it then follows that $f(x) \in R(0)$. $\underline{f \text { satisfies PA2 }}$. For all $x, y \in \mathbb{R}^{d}$, it follows from A2 that $R(f(x))+R(f(y))=\tilde{R}(x)+\tilde{R}(y) \subset \tilde{R}(x+y)=R(f(x+y))$. From A4, this provides that $R(0) \subset\{-f(x+y)+f(x)+f(y)\}+R(0)$. Since $0 \in R(0)$ by A0, this proves that $f(x+y)-f(x)-f(y) \in R(0)$. $\underline{f \text { satisfies PA3 }}$. For all $t>0$ and $x \in \mathbb{R}^{d}$, it follows from A3 and A4 that $\{-t f(x)\}+R(0)=t R(f(x))=t \tilde{R}(x)=\tilde{R}(t x)=R(f(t x))$ $=\{-f(t x)\}+R(0)$. Since $0 \in R(0)$ by A0, this provides that $f(t x)-t f(x) \in R(0) \cap-R(0)$ $=\vec{R}_{0} . \quad \underline{f \text { satisfies PA4 }}$. For all $x \in \mathbb{R}^{n}$ and $y \in \mathbb{R}^{d}$, it follows from A4 that $\tilde{R}(\bar{x}+y)$ $=\{-x\}+\tilde{R}(y)=\{-x\}+R(f(y))=\{-x-f(y)\}+R(0)$. On the other hand, one has $\tilde{R}(\bar{x}+y)=R(f(\bar{x}+y))=\{-f(\bar{x}+y)\}+R(0)$. Thus, since $0 \in R(0)$, we obtain that $f(\bar{x}+y)-x-f(y) \in \vec{R}_{0}$. 2. Conversely, we assume that $f$ is an $R$-coherent portfolio aggregator, and that the $(n, n)$ - coherent risk measure $R$ satisfies $\mathrm{A} 1_{s}$. By Lemma ??, $\tilde{R}=$ $R \circ f$ is a well-defined set-valued map from $L_{d}^{\infty}$ into $\mathbb{R}^{n}$. Observe that a similar argument to that used in the proof of Property ?? shows that $\mathrm{A} 1_{s}$ is equivalent to the following statement :

$$
\text { For all } X, Y \in L_{d}^{\infty}: X-Y \in R(0) P-\text { a.s. } \quad \Longrightarrow \quad R(Y) \subset R(X) \text {. }
$$

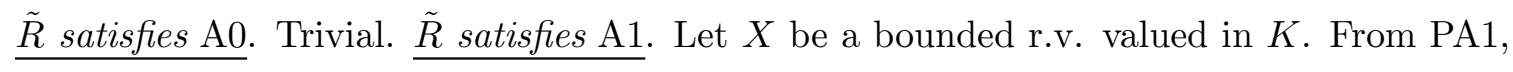
we have $f(X) \in R(0) P$-a.s. and therefore

$$
R(0) \subset R(f(X))=\tilde{R}(X)
$$

by condition $\mathrm{A} 1_{s}$. Now, from PA3, it follows that $f(0) \in \vec{R}_{0}$. Then, $\tilde{R}(0)=R(f(0))=$ $\{-f(0)\}+R(0) \subset R(0)$ since $-f(0) \in R(0)$. In view of (??), this proves that $\tilde{R}(0) \subset \tilde{R}(X)$. 


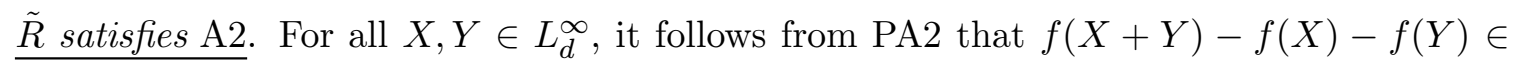
$R(0) P$-a.s. Since $R$ is an $(n, n)-$ coherent risk measure, This implies that $\tilde{R}(X)+\tilde{R}(Y)=$ $R(f(X))+R(f(Y)) \subset R(f(X)+f(Y)) \subset R(f(X+Y))=\tilde{R}(X+Y)$ by (??). $\underline{\tilde{R} \text { satisfies A3. }}$ For all $t>0$ and $X \in L_{d}^{\infty}$, Property 3 says that $f(t X)-t f(X) \in R(0)$ and $t f(X)-f(t X) \in$ $R(0) \mathbb{P}$-a.s. From (??), this implies that $\tilde{R}(t X)=R(f(t X))=R(t f(X))=t R(f(X))=$ $t \tilde{R}(X)$ since $R$ is an $(n, n)$ - coherent risk measure. $\underline{\tilde{R} \text { satisfies } \mathrm{A} 4}$. For all $x \in \mathbb{R}^{n}$ and $X \in L_{d}^{\infty}$, it follows from PA4 that $f(X+\bar{x})-f(X) \overline{-x \in \vec{R}_{0}=}-R(0) \cap R(0) \mathbb{P}$-a.s. Using again (??) twice, this proves that $\tilde{R}(X+\bar{x})=\{-x\}+\tilde{R}(X)$.

Example 6.2 (Coherent linear portfolio aggregators) Suppose that $\vec{R}_{0}=\{0\}$, and let $f(y)$ $:=A y$ where $A$ is a $(n, d)$ matrix. Clearly, $f$ satisfies conditions PA2 and PA3. A necessary and sufficient condition for $f$ to satisfy condition PA4 is that the matrix $A$ be of the form :

$$
A=\left(I_{n} \mid B\right) \quad \text { where } I_{n} \text { is the identity matrix in } \mathbb{R}^{n} .
$$

Hence $f$ is an $R$-coherent portfolio aggregator if and only if $A K \subset R(0) . \underline{\mathrm{n}=1}$ : in this case, $R(0)=K_{1}=\mathbb{R}_{+}, A=\left(1, b_{n+1}, \ldots, b_{d}\right)$ with $b_{j} \in \mathbb{R}$ for all $j=n+1, \ldots, d$. The condition $A K \subset R(0)$ says that $A x \geq 0$ for all $x \in K$. Hence $f$ is an $R$-coherent portfolio aggregator of $A \in K_{1}^{\circ}:=\left\{y \in K^{\circ}: y^{1}=1\right\}$.

Example 6.3 (Coherent nonlinear portfolio aggregators, $d=2, n=1$ ) Again, we have $R(0)=K_{1}=\mathbb{R}_{+}$, and therefore $\vec{R}_{0}=\{0\}$. Then, it is easily checked that Conditions PA1,PA3 and PA4 imply that $f$ is of the form :

$$
f(x)=x a_{+} \mathbf{1}_{\{x \geq 0\}}+x a_{-} \mathbf{1}_{\{x \leq 0\}}
$$

where $a+=\left(1, \alpha_{+}\right) \in K_{+}^{\circ}, a_{-}=\left(1, \alpha_{-}\right) \in K_{-}^{\circ}$ with $K_{+}=K \cap\left(\mathbb{R} \times \mathbb{R}_{+}\right)$and $K_{-}=$ $K \cap\left(\mathbb{R} \times \mathbb{R}_{-}\right)$. Now Condition PA2 says that $f$ is concave, i.e. $\alpha_{+} \leq \alpha_{-}$. Hence $f$ is an $R$-coherent portfolio aggregator if and only if

$$
a_{+}=\left(1, \alpha_{+}\right) \text {and } a_{-}=\left(1, \alpha_{-}\right) \in K^{\circ} \text { with } \quad \alpha_{+} \leq \alpha_{-}
$$

Example 6.4 (liquidation function, $n=1$ ) Let $R$ be a $(1,1)$-coherent risk measure. then the liquidation function $\ell$ defined in (??) is an $R$-coherent portfolio aggregator.

Remark 6.3 The function $\pi$ defined in (??) is not a portfolio aggregator in general. To see this, consider the case $d=3, n=2$, and $K$ defined as in Example ?? with proportional transaction costs matrix

$$
\lambda=\alpha\left(\begin{array}{lll}
1 & 1 & 1 \\
1 & 1 & 1 \\
1 & 0 & 1
\end{array}\right) \text { for some } \alpha>0
$$


Then, it is easily checked that $x:=(0,1,-1) \in K$, and $\bar{\pi}(x)=(-1-\alpha, 1,0) \in-K$. Since $K \cap-K=\{0\}$, it follows from Property ?? that $R(0) \cap-K_{2}=\{0\}$, and therefore $\pi(x) \notin$ $R(0)$, for all $(2,2)-$ coherent risk measure. Hence there is no $(2,2)-$ coherent risk measure for which $\pi$ is a coherent portfolio aggregator.

\section{Coherent aggregation of risk}

Definition 7.1 Let $R$ be a $(d, d)$-coherent risk measure. A function $g: \mathbb{R}^{d} \longrightarrow \mathbb{R}^{n}$ is an $R$-coherent risk aggregator if : RA1 $g(R(0)) \neq \mathbb{R}^{n}$ and $0 \in g(R(0))$; RA2 For all $x, y \in \mathbb{R}^{d}: g(x)+g(y) \in \operatorname{cl}[g(R(-x-y))]$; RA3 For all $x \in \mathbb{R}^{d}$ and $t>0$ :

$$
g(t x) \in \operatorname{cl}[t g(R(-x))] \quad \text { and } \operatorname{tg}(x) \in \operatorname{cl}[g(t R(-x))] ;
$$

RA4 For all $x \in \mathbb{R}^{n}$ and $y \in \mathbb{R}^{d}$ :

$$
g(\bar{x}+y) \in x+\operatorname{cl}[g(R(-y))] \quad \text { and } \quad x+g(y) \in \operatorname{cl}[g(R(-\bar{x}-y))] .
$$

Some examples of coherent risk aggregators will be discussed at the end of this section.

Theorem 7.1 Let $R$ be a $(d, d)$-coherent risk measure, and let $g$ be a mapping from $\mathbb{R}^{d}$ into $\mathbb{R}^{n}$. Define the set-valued map

$$
\begin{aligned}
\operatorname{cl}[g \circ R]: L_{d}^{\infty} & \longrightarrow \mathbb{R}^{n} \\
X & \longmapsto \operatorname{cl}[g(R(X))] .
\end{aligned}
$$

Then, $\operatorname{cl}[g \circ R]$ is a $(d, n)$-coherent risk measure if and only if $g$ is an $R$-coherent risk aggregator.

Proof. Set $\tilde{R}:=\operatorname{cl}[g \circ R]$. 1. Suppose that $\tilde{R}$ is a coherent risk measure, and let us prove that $g$ satisfies properties RA. $g$ satisfies RA1. Immediate, since $0 \in \tilde{R}(0)$ by A0. $\underline{g \text { satisfies } \mathrm{RA} 2}$. For all $x, y \in \mathbb{R}^{d}$, it follows from A2 that $\tilde{R}(-x)+\tilde{R}(-y) \subset \tilde{R}(-x-y)$. By A4, this means that $\operatorname{cl}[g(\{x\}+R(0))]+\operatorname{cl}[g(\{y\}+R(0))] \subset \operatorname{cl}[g(R(-x-y))]$, and the required result follows from the fact that $0 \in R(0)$. g satisfies $\mathrm{RA} 3$. For all $x \in \mathbb{R}^{d}$ and $t>0$, it follows from A3 that $\tilde{R}(-t x)=t \tilde{R}(-x)$. By A4, this means that $\operatorname{cl}[g(\{t x\}+$ $R(0))]=t \operatorname{cl}[g(R(-x))]$, which provides $g(t x) \in t \operatorname{cl}[g(R(-x))]$. This also can be written in $t \mathrm{cl}[g(\{x\}+R(0))]=\operatorname{cl}[g(t R(-x))]$, which provides the remaining property. $\underline{g \text { satisfies RA } 4 .}$ For all $x \in \mathbb{R}^{n}$ and $y \in \mathbb{R}^{d}$, it follows from A4 that $\tilde{R}(-\bar{x}-y)=\{x\}+\tilde{R}(-y)$, i.e. $\operatorname{cl}[g(\{\bar{x}+y\}+R(0))]=\{x\}+\operatorname{cl}[g(\{y\}+R(0))]$, and the result follows again from the fact that $0 \in R(0)$. 2. Conversely, let $g$ be an $R$-coherent risk aggregator, and let us prove that $\tilde{R}$ satisfies conditions A. $\tilde{R}$ satisfies A0. Immediate from the fact that $R$ satisfies A0, the 
definition of $\tilde{R}$, and RA1. $\tilde{R}$ satisfies A1. Let $X \in L_{d}^{\infty}$ be such that $X \succeq 0 P$-a.s. Then,

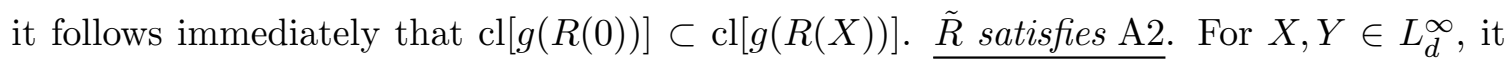
follows from the coherency of $R$ that $R(X)+R(Y) \subset R(X+Y)$. Then, it follows immediately that $g(R(X)+R(Y)) \subset g(R(X+Y))$. By RA2, this provides $\operatorname{cl}[g(R(X))]+\operatorname{cl}[g(R(X))]$

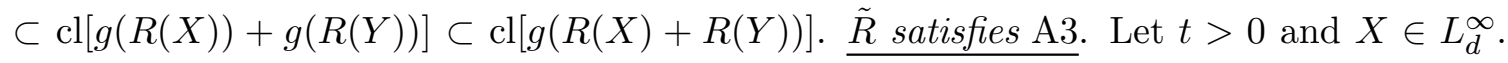
Since $R$ is coherent, we have $g(R(t X))=g(t R(X)) \subset t \operatorname{cl}[g(R(X))]$ by RA3. Therefore $\operatorname{cl}[g(R(t X))] \subset t \operatorname{cl}[g(R(X))]$. Conversely, consider some $x \in R(X)$. By the self-consistency Property ??, $R(0) \subset R(X+x)$. Then by RA3, $t g(x) \in \operatorname{cl}[g(R(-t x))]=\operatorname{cl}[g(\{t x\}+t R(0))]$ $\subset \operatorname{cl}[g(\{t x\}+t R(X+x))]=\operatorname{cl}[g(R(t X))]$. From the arbitrariness of $x \in R(0)$, this proves

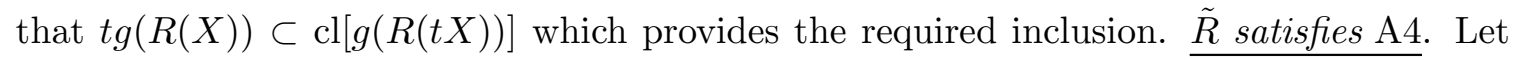
$X \in L_{d}^{\infty}$ and $a \in \mathbb{R}^{n}$. Since $R$ is coherent, we have $g(R(X+\bar{a}))=g(\{-\bar{a}\}+R(X))$. Now, for any $x \in R(X)$, we have $R(0) \subset R(X+x)$ by the self-consistency Property ??. By use of RA4, this provides $g(\{-\bar{a}\}+x) \in\{-a\}+\operatorname{cl}[g(R(-x))]=\{-a\}+\operatorname{cl}[g(\{x\}+R(0))] \subset$ $\{-a\}+\operatorname{cl}[g(\{x\}+R(X+x))]=\{-a\}+\operatorname{cl}[g(R(X))]$. By the arbitrariness of $x \in R(X)$, this proves that $g(\{-\bar{a}\}+R(X)) \subset\{-a\}+\operatorname{cl}[g(R(X))]$. Conversely, using again RA4, we see that $-a+g(x) \in \operatorname{cl}[g(R(\bar{a}-x))]=\operatorname{cl}[g(\{-\bar{a}+x\}+R(0))] \subset \operatorname{cl}[g(\{-\bar{a}+x\}+R(X+x))]$ $=\operatorname{cl}[g(\{-\bar{a}\}+R(X))]$. By the arbitrariness of $x \in R(X)$, this shows that $\{-a\}+g(R(X))$ $\subset \operatorname{cl}[g(\{-\bar{a}\}+R(X))]$.

Example 7.1 (Risk liquidation) Let $\pi$ be the function defined in (??-??-??) by means of the liquidation function $\ell$, and define

$$
g(x):=-\pi(-x) \text { for all } x \in \mathbb{R}^{d}
$$

Then $g$ is an $R$-coherent risk aggregator for all $(d, d)$ - coherent risk measure $R$. Indeed : - Since $0 \in R(0)$, it follows that $0=g(0) \in g(R(0))$. Now let $-y$ be any vector in the interior of $-K_{n}=\left\{x \in \mathbb{R}^{n}: \bar{x} \in-K\right\}$. Suppose that $g(R(0))=\mathbb{R}^{n}$ and let us work towards a contradiction. Then, $-\bar{y}=-\bar{\pi}(-x)$ for some $x \in R(0)$. By definition of $\bar{\pi}$, this implies that $-\bar{y} \succeq x \in R(0)$ and therefore $-\bar{y} \in R(0)$ by Property ??. Since $\bar{y} \in \operatorname{int}(K)$, the required contradiction follows from Property ??. Hence $g$ satisfies RA1. - From the definition of $\bar{\pi}$, it follows that $-\bar{\pi}(-x)-\bar{\pi}(-y) \succeq-\bar{\pi}(-x-y)$ for all $x, y \in \mathbb{R}^{d}$. This means that $-\bar{\pi}(-x)-\bar{\pi}(-y)=-\bar{\pi}(-x-y)+\bar{z}=-\bar{\pi}(-x-y-\bar{z})$ for some $z \in K_{n}$. Now since $K_{n}$ $\subset R(0)$, this provides that $-\bar{\pi}(-x)-\bar{\pi}(-y) \in-\bar{\pi}(-x-y-R(0))=-\bar{\pi}(-R(-x-y))$ by the translation invariance axiom A4. hence $g$ satisfies RA2. - We leave the verification of Properties RA3 and RA4 for the reader, since it can be done easily by similar arguments. 


\section{References}

[1] C.D. Aliprantis And K.C. Border (1999). Infinite dimensional analysis, SpringerVerlag.

[2] Ph. Artzner, F. Delbaen, J.-M. Eber and D. Heath (1998). Coherent Measures of Risk, Finance and Stochastics 9, 203-228.

[3] F. Delbaen (2000). Coherent risk measures on general probability spaces, Preprint.

[4] N. Dunford And J. Schwartz (1958). Linear operators, Vol I, Interscience.

[5] A. Grothendieck (1973). Topological vector spaces, Gordon and Breach, New York.

[6] Y. Kabanov (1999). Hedging and liquidation under transaction costs in currency markets, Finance and Stochastics 3, 237-248.

[7] Y. Kabanov and C. Stricker (2000). The Harrison-Pliska arbitrage Theorem under transaction cost, Journal of Mathematical Economics, to appear.

[8] R.T. Rockafellar (1997). Convex Analysis, Princeton Landmarks in Mathematics, Princeton University Press.

[9] K. Yosida And E. Hewitt (1952). Finitely additive measures, Trans. Amer. Math. Soc. 72, 46-66. 